\title{
China and Germany join forces over SARS
}

Alison Abbott, Munich

When Jiang Hua-Liang and Rolf Hilgenfeld ran into each other in the empty corridors of a Beijing hotel earlier this month, they immediately discovered how much they had to talk about.

Now they are hoping to work together to study the virus believed to be behind severe acute respiratory syndrome (SARS). The planned project is just one example of a host of unexpected international alliances that have sprung up in the wake of the mystery disease.

Jiang heads the Drug Discovery and Design Center at the Shanghai Institute of Materia Medica, and Hilgenfeld is a structural biologist at the University of Lübeck in Germany. The pair were participating in a workshop in the Chinese capital on 3-4 June, organized by the Sino-German Centre for Science Promotion to encourage collaboration between SARS researchers.

The German delegation of 14 virologists was the first of its type to arrive in China since the SARS epidemic began, and it arrived to find that fear of the disease had emptied Beijing's streets of residents and tourists.

Hilgenfeld is an expert in coronaviruses, the family to which the SARS virus belongs, and has detailed crystal structures of a key SARS virus protein. Jiang uses supercomputers for the mass screening of drug candidates — identifying molecules that fit snugly into computerized three-dimensional models of proteins. The researchers realized how efficiently they could work together to develop new leads for anti-SARS drugs.

The idea is one of a dozen or so that over the next two months will be turned into formal funding proposals for the Sino-German Centre, which is jointly financed to the tune of $€ 2$ million (US\$2.4 million) annually by research agencies in Germany and China.

The German visitors say that they were taken aback to see how far and fast Chinese scientists have come since turning their attention to the SARS virus a couple of months ago.

China has had thousands of SARS cases and, consequently, has a wealth of patient material and clinical experience that the German scientists - who only have isolates from two clinical cases that passed through Frankfurt airport in March — want to access.

"Benefits of the collaborations cut both ways," says Hans-Dieter Klenk, head of Marburg University's Institute of Virology, who led the delegation. "Germany has good basic research experience with coronaviruses." It also has important tools such as a new-generation polymerase chain reaction (PCR) test - a method used to amplify selected regions of DNA — which is waiting to be tested in patients.
But the German researchers say that the Chinese scientists are strongly possessive of their virus isolates. They insist that it would be too dangerous to send them to Germany, and that any collaborative work should therefore be done in China. "They are understandably concerned not to lose control of their resources," says Klenk.

The Germans add that they are happy to work in China on clinical questions such as the immune response to the virus, which will benefit from the hundreds of different isolates the Chinese have, scores of which have already been fully sequenced. Christian Drosten, a clinical virologist at the Bernhard Nocht Institute for Tropical Medicine in Hamburg, says that he is also optimistic about potential collaborations to put his PCR test through its paces, but says that he realizes it may not be so easy in practice.

Some of the potential collaborations will address more basic research questions, such as understanding how the virus regulates its own gene expression, which can be done without working on the entire virus.

The visitors were also struck by the high

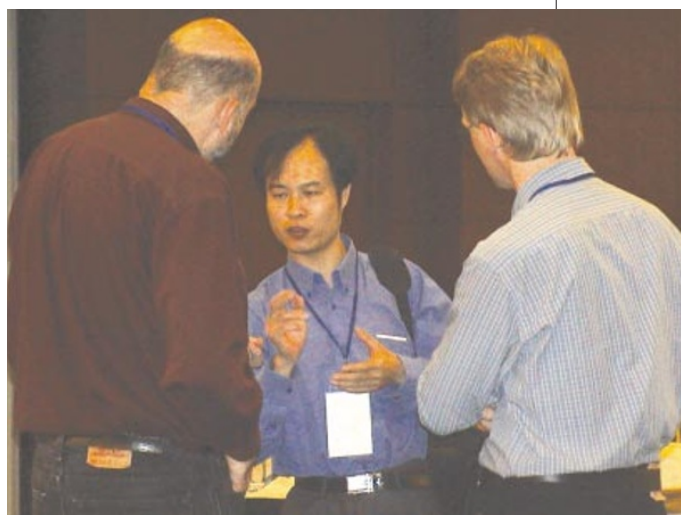

Common goal: Jiang Hua-Liang (centre) explores collaboration with German researchers in Beijing.

laboratory standards, and the commitment of the Chinese scientists. "I was really surprised to see so much investment in high-tech laboratory equipment," says Hilgenfeld, who paid a late-night visit to Tsinghua University in Beijing and found droves of researchers still beavering away at 11 o'clock. "You would never see that in Germany," he says.

\section{Clinical data marshalled to treat HIV}

\section{Tom Clarke, London}

A database is set to help doctors treat HIV. It attempts to identify the best drug combinations to keep a patient's infection in check, on the basis of genetic sequences of drug-resistant strains of the virus.

The international collaboration that is developing the system - the HIV Resistance Response Database Initiative (RDI) unveiled its latest results on 12 June at the 12th International HIV Drug Resistance Workshop in Los Cabos, Mexico. And although the system isn't the first to deduce information about the drug resistance of particular HIV strains, it is the first to attempt to do so on such a scale, and to be based on clinical results.

"We're aiming to produce tools that can predict treatment response in actual patients," says Brendan Larder, a virologist who chairs the initiative's core group. The system could eventually be available to all physicians online, enabling them to use a patient's HIV gene sequence to help to predict what drug treatment options would be most successful.

Rapidly mutating HIV strains readily develop resistance to drugs. Up to half of all patients living with HIV must switch drug combinations at least once to control their viral load. Studies have identified about 200 mutations associated with resistance to the 16 drugs commonly given to control the virus.

Doctors already use computer models to establish the optimal combination of two or three drugs that should be given to treat a particular, resistant strain of HIV. But that is an inexact business, given that a patient's virus may harbour as many as $\mathbf{4 0}$ drugresistance mutations. Also, input to the existing computer models usually derives from the ability of different strains to survive in test-tube experiments - which may not reflect the outcomes in patients.

"What's missing is a link to clinical response," says Joep Lange, director of the National AIDS Therapy Evaluation Center in Amsterdam. "At the moment, a lot of mistakes are being made." Because the RDI software 'learns' from real patient data, it could surmount these problems, he says.

The latest test of the software showed that the system could correctly predict which drug combinations would bring HIV under control, and by how much, $78 \%$ of the time.

This isn't reliable enough for clinical use, Larder admits. But the latest results are from the 750 cases in the original database. And data from about 4,000 patients at AIDS treatment centres in Europe and North America are currently being added. Once that happens, "we hope to achieve $90 \%$ accuracy", says Larder. 